\title{
Pancreatic Cysts after Endoscopic Ultrasonography-Guided Ethanol and/or Paclitaxel Ablation Therapy: Another Mimic of Pancreatic Pseudocysts
}

\author{
Soyeon $\mathrm{An}^{\mathrm{a}}$ You-Na Sung ${ }^{\mathrm{b}}$ Sung Joo Kim ${ }^{\mathrm{b}}$ Dong-Wan Seo ${ }^{\mathrm{c}}$ \\ Sun-Young Jun ${ }^{a}$ Seung-Mo Hong ${ }^{b}$ \\ aDepartment of Pathology, Incheon St. Mary's Hospital, College of Medicine, The Catholic University of Korea, Seoul, \\ Republic of Korea; 'bepartment of Pathology, Asan Medical Center, University of Ulsan College of Medicine, Seoul, \\ Republic of Korea; 'Department of Gastroenterology, Asan Medical Center, University of Ulsan College of Medicine, \\ Seoul, Republic of Korea
}

\section{Keywords}

Endoscopic ultrasonography · Ablation · Pancreas .

Mucinous cystic neoplasm · Pseudocyst

\section{Abstract}

Background: Endoscopic ultrasound-guided ablation (EUSA) therapy is a minimally invasive procedure for pancreaticcystic tumors in patients with preoperative comorbidities or in patients who are not indicated for surgical resection. However, histopathologic characteristics of pancreatic cysts after ablation have not been well-elucidated. Methods: Here, we analyzed pathological findings of 12 surgically resected pancreatic cysts after EUS-A with ethanol and/or paclitaxel injection. Results: Mean patient age was $49.8 \pm 13.6$ years with a 0.3 male/female ratio. Clinical impression before EUS-A was predominantly mucinous cystic neoplasms. Mean cyst size before and after ablation therapy was similar $(3.7 \pm 1.0 \mathrm{~cm}$ vs. $3.4 \pm 1.6 \mathrm{~cm} ; p=0.139$ ). Median duration from EUS-A to surgical resection was 18 (range, 1-59) months. Mean percentage of the residual neoplastic lining epithelial cells were $23.1 \pm 37.0 \%$. Of the resected cysts, 8 cases (67\%) showed no/ minimal $(<5 \%)$ residual lining epithelia, while the remaining 4 cases (33\%) showed a wide range of residual mucinous ep-

karger@karger.com www.karger.com/pat

Karger $\stackrel{\text { ! }}{\div}$
(C) 2021 The Author(s)

Published by S. Karger AG, Basel

This is an Open Access article licensed under the Creative Commons Attribution-NonCommercial-4.0 International License (CC BY-NC) (http://www.karger.com/Services/OpenAccessLicense), applicable to the online version of the article only. Usage and distribution for commercial purposes requires written permission. ithelia (20-90\%). Ovarian-type stroma was noted in 5 cases (42\%). Other histologic features included histiocytic aggregation (67\%), stromal hyalinization (67\%), diffuse egg shelllike calcification along the cystic wall (58\%), and fat necrosis (8\%). Conclusion: Above all, diffuse egg shell-like calcification along the pancreatic cystic walls with residual lining epithelia and/or ovarian-type stroma were characteristics of pancreatic cysts after EUS-A. Therefore, understanding these histologic features will be helpful for precise pathological diagnosis of pancreatic cystic tumor after EUS-A, even without knowing the patient's history of EUS-A.

(c) 2021 The Author(s) Published by S. Karger AG, Basel

\section{Introduction}

Pancreatic cysts encompass a wide range of lesions, from nonneoplastic cysts to precursor lesions of pancreatic ductal adenocarcinomas [1]. Owing to recent technical advances in imaging modalities, such as computed tomography $(\mathrm{CT})$ scan and magnetic resonance imaging (MRI), the detection of pancreatic cysts is continuously

Sun-Young Jun and Seung-Mo Hong are co-corresponding authors.
Correspondence to:

Sun-Young Jun, drssun@ catholic.ac.kr Seung-Mo Hong, smhong28@ gmail.com 
increasing [2-5]. Based on the results of a meta-analysis, which included 17 studies and 48,860 patients, the prevalence of incidentally detected pancreatic cysts is $8 \%$ [6]. Pancreatic cysts can be classified as nonneoplastic, benign (serous cystadenomas [SCAs]), cyst with malignant-potential, and cystic degeneration of malignant tumors (cystic degeneration of pancreatic ductal adenocarcinomas, neuroendocrine tumors, pancreatoblastoma, acinar cell carcinomas, and solid pseudopapillary neoplasms [SPNs]) [6]. Nonneoplastic pancreatic cysts contain pseudocysts, ductal retention cysts, congenital cysts, and endometrioid cysts [6]. Serous cystic neoplasms (also known as SCAs) are benign neoplasms. Pancreatic cysts with malignant-potential can be further classified as intraductal papillary mucinous neoplasms and mucinous cystic neoplasms (MCNs) [4, 7]. In addition, SPNs are, in general, malignant solid neoplasms in which degree of cystic degeneration increases with tumor size [8]. Because they have to balance their risk management of disease progression for cysts with malignant potential and avoid overtreatment for nonneoplastic or benign neoplastic cysts, management of pancreas cysts is complicated for clinicians.

Surgical resection of pancreatic cystic neoplasms is associated with substantially increased morbidity rates of $20-40 \%$ and mortality rates up to $2 \%$ [9-11]. In addition, preoperative diagnosis of pancreatic cysts by CT scan may not be correct, since only one-quarter of the surgically resected serous cystic neoplasms s were correctly diagnosed by preoperative CT scan in a large-scale study $[12,13]$. Recently, endoscopic ultrasound (EUS)-guided ablation (EUS-A) therapy was introduced as a promising treatment option for patients with pancreatic cysts using minimally invasive approaches [7, 13-15]. Ethanol was the most commonly used ablative agent [16]. In addition, booster injections of chemotherapeutic agents improved the ablative effects of ethanol owing to a synergistic effect of lysis of cystic lining epithelial cells by a combination of ethanol lavage and secondary paclitaxel injection $[14,15]$. The complete resolution rates after EUS-A therapy ranged from $9 \%$ to $85 \%$ across several studies [13-15, 17-26]. In the literature, a few histologic features of EUS-A therapy were reported after surgical resection [13, 14, 20, 24-26], including denudation of the epithelial cells, atrophy, and fibrosis $[14,20,24]$. However, histopathologic characteristics of pancreatic cysts after ablation have not been comprehensively evaluated. In this study, we systemically analyzed the histopathologic characteristics of surgically resected pancreatic cysts after EUS-guided ethanol and/or paclitaxel ablation therapy.

\section{Materials and Methods}

This study was approved from the Institutional Review Board (approval number, 2013-0618) with waiver of patient's consents. Twelve cases of surgically resected pancreatic cysts after EUS-A with ethanol and/or paclitaxel injection, from 2006 to 2015, were selected from the pathology database. Inclusion and exclusion criteria for EUS-A therapy were the same, as previously reported [14]. In brief, the inclusion criteria were as follows: (1) unilocular or oligolocular pancreatic cysts; (2) indeterminate pancreatic cysts for which EUS-guided fine-needle aspiration was indicated to obtain additional information; and/or (3) pancreatic cysts with increased cyst size during the follow-up period. The exclusion criteria for EUS-A therapy were as follows: (1) pancreatic cysts that had typical SCA morphology (honeycomb appearance); (2) a recently identified episode of pancreatitis and parenchymal changes on imaging which suggested a pseudocyst; (3) the presence of communication between the cystic lesion and the main pancreatic duct on endoscopic retrograde cholangiopancreatography; (4) overt carcinomas with peripancreatic invasion by radiologic imaging; and/or (5) patients with a bleeding tendency (prothrombin time $>1.5$ times the international normalized ratio or a platelet count $<50,000 / \mu \mathrm{L})$. Clinical characteristics were obtained from the electronic medical records, which included age, sex, imaging findings, CEA levels of serum and cystic fluid, and outcomes after the ablation therapy.

Hematoxylin and eosin-stained slides from pancreatic cysts (mean, $7.3 \pm 4.2$ slides) were selected and reviewed by 2 pathologists (S.A. and S.-M.H.). Evaluated histopathologic features included the presence of residual lining epithelia of the entire pancreatic cysts, presence of residual ovarian-type stroma, inflammatory cell infiltrations, hyalinization, calcification, hemorrhage, pigmentation, cholesterol cleft, and fat necrosis.

Statistical analyses were performed with SPSS 20.0 (IBM SPSS, Armonk, NY, USA). The Wilcoxon signed rank test was used to compare the size of pancreatic cysts on initial and follow-up CT scan images. A $p$ value of $<0.05$ was considered to be statistically significant.

\section{Results}

\section{Characteristics of the Cases}

Patient's clinicopathologic features are summarized in Table 1. Mean patient age was $49.8 \pm 13.6$ years with a $0.3 \mathrm{male} / \mathrm{female}$ ratio. Pretreatment imaging diagnoses before EUS-A included the following: $10 \mathrm{MCNs}(84 \%)$, one SCA ( $8 \%$ ), and one epidermoid cyst (8\%). Mean cystic fluid CEA and amylase levels were $1563.8 \pm 2682.3 \mathrm{ng} /$ $\mathrm{mL}$ (range, $1.4-8,190 \mathrm{ng} / \mathrm{mL}$ ) and 1186.6 (range, 11$6,005) \mathrm{U} / \mathrm{L}$, respectively. The mean size of pancreatic cysts before ablation therapy was $3.7 \pm 1.0 \mathrm{~cm}$. On the first follow-up CT scan imaging after ablation therapy, the mean size of pancreatic cysts was $3.4 \pm 1.6 \mathrm{~cm}$. Compared to the mean size before ablation therapy, mean size after ablation was not different $(p=0.139)$. Median sec- 
ond follow-up time after ablation was 18 (range, 1-59) months. The mean size of pancreatic cysts after ablation therapy at the second follow-up was $3.6 \pm 1.7 \mathrm{~cm}$. On the second follow-up CT scan imaging, compared to the size before ablation therapy, partial resolution (at least a 30\% decrease in the longest diameter) was observed in 4 cases. Meanwhile, the remaining 8 cases $(67 \%)$ had persistent cysts. Among those persistent cysts, 2 cases $(2 / 8,25 \%)$ were progressive with at least a $20 \%$ increase in the longest diameter of the cyst size. In addition, leakage of cystic fluid was observed in 2 cases (17\%). Finally, all 12 patients underwent surgical resection: 9 underwent distal pancreatectomy (75\%); 2 (17\%) underwent pylorus preserving pancreatoduodenectomy; and 1 (8\%) underwent central pancreatectomy.

\section{Histopathologic Findings}

Representative images of surgically resected pancreatic cysts after ablation therapy during gross examination are shown in Figure 1. Median time between ablation therapy and surgical resection of pancreatic cysts was 18 (range, 1-59) months.

Mean percentage of residual lining epithelial cells of the pancreatic cysts after surgical resection were $23 \pm 37 \%$ (Fig. 2a). Eight cases $(67 \%)$ showed either no $(n=6)$ or $<5 \%(n=2)$ residual lining epithelia. In contrast, 4 cases (33\%) showed various degrees of residual mucinous epithelium (mean, $67.5 \pm 33.0$; range, $20-90 \%$ ). In addition, ovarian-type stroma was observed in 5 cases $(42 \%$, Fig. 2a). Five cases had both residual lining mucinous epithelia and ovarian-type stroma, which therefore could be diagnosed as MCNs.

The remaining 7 cases which had no or minimal residual lining epithelia had been diagnosed as a pseudocyst $(5 / 12,42 \%)$, a degenerated cyst $(1 / 12,8 \%)$ or a hemorrhagic cyst $(1 / 12,8 \%)$ in the original pathology reports without providing information about preoperative ablation treatment at the time of pathology diagnoses. Frequently observed histologic features include moderate chronic active stromal inflammation (9/12, 75\%, Fig. 2b), aggregation of foamy histiocytes (8/12, 67\%, Fig. 2c), marked stromal hyalinization $(8 / 12,67 \%)$, stromal fibrosis $(5 / 12,42 \%)$, and diffuse calcification along the cystic wall (7/12, 58\%, Fig. 2d). Less frequently, necrotic tissue with brown pigments and numerous neutrophilic infiltration $(3 / 12,25 \%$, Fig. 3a), cholesterol clefts $(3 / 12,25 \%$, Fig. $3 b)$, granulation tissue $(2 / 12,8 \%)$, hemorrhage $(2 / 12$, $8 \%$, Fig. 3 c), and fat necrosis $(2 / 12,8 \%$, Fig. $3 d)$ were observed.

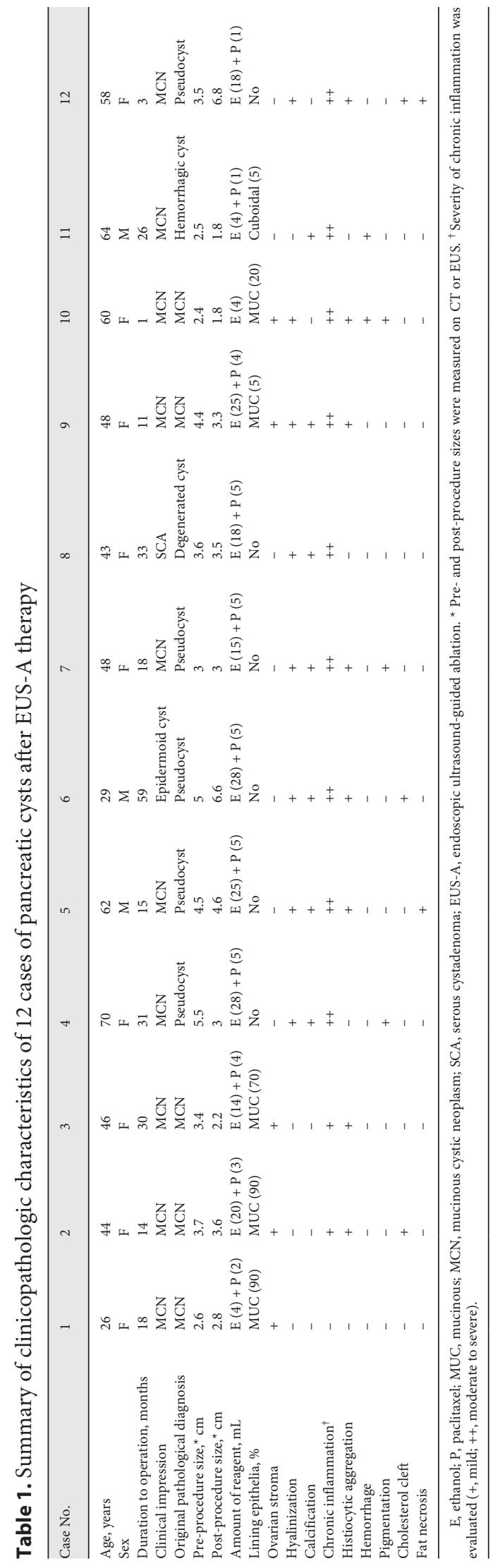



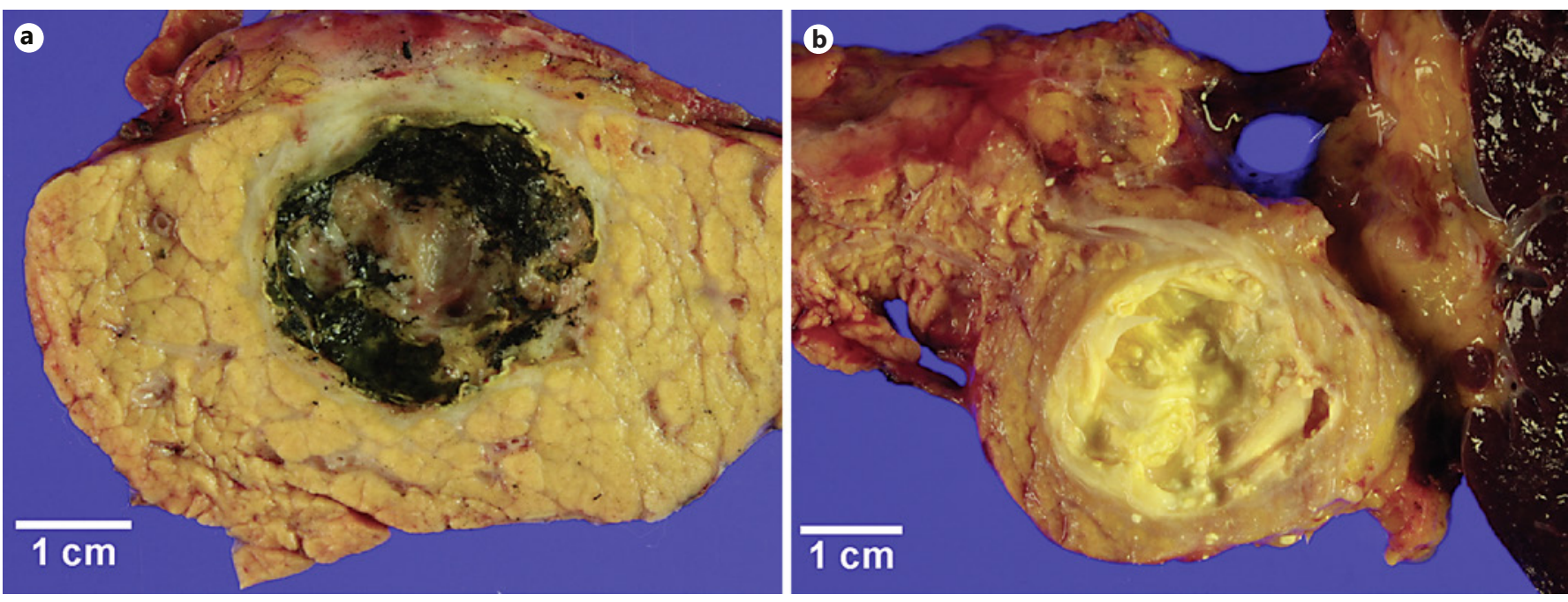

Fig. 1. a, b Representative gross images of pancreatic cysts after endoscopic ultrasound-guided ablation therapy. Yellow diffuse egg shell-like calcifications are along the pancreatic cystic walls.

\section{Discussion}

EUS-A therapy has been developed as a minimally invasive approach, and ethanol injection was the most commonly used ablative agent, which lead to cell death by lysis of cytoplasmic membrane, protein denaturation, and vascular occlusion [16]. In addition, paclitaxel improved the ablative effects of the therapy by inhibiting microtubule function during cell division [27], and increasing the synergistic effect after ethanol injection $[14,15]$. The hydrophobic nature of paclitaxel increases its period of action within the pancreatic cysts [15]. Several previous studies have focused on the efficacy and safety of EUS-A therapy of pancreatic cysts using ethanol and/or paclitaxel [13-15, 17-26]. In this study, we focused on histopathologic aspects of this ablation therapy.

We quantitatively evaluated residual neoplastic lining epithelial cells and found that about two-thirds (8/12, $67 \%$ ) had no or minimal residual lining epithelial cells in surgically resected pancreatic cysts after ablation. Similar to our results, Oh and colleagues [14] reported that $75 \%$ $(3 / 4)$ of the cases showed marked epithelial ablation (range, 25-100\%) in resected pancreatic cysts after ablation. In contrast, in nontreated MCN cases before surgical resection, approximately half of the neoplastic epithelial lining was eroded [28]. Therefore, pancreatic cysts treated with EUS-guided ablation therapy may have a wider area of the cystic wall without covering lining epithelial cells after ablation.
In present study, $84 \%(10 / 12)$ of cases had a clinical impression of pancreatic cysts, mainly MCNs based on pretreatment imaging studies. As is well-known, ovariantype stroma is a distinct histologic features of MCNs [28]. As expected, we found ovarian-type stroma in about half $(5 / 12,42 \%)$ of the cases, and all 5 cases with ovarian-type stroma had residual lining mucinous epithelia. On the other hand, among the remaining 7 cases without ovarian-type stroma, 6 cases $(6 / 7,86 \%)$ showed complete epithelial ablation. However, in a study by Gan and colleagues [20], ovarian-type stroma was found in all resected pancreatic cysts after EUS-guided ethanol lavage $(n=$ 5). Moreover, they found ovarian-type stroma even in a totally denuded pancreatic cyst [20]. The discrepancy in these results regarding residual ovarian-type stroma and residual lining epithelia might be caused by different types or injected amounts of ablative agents. Although pre-ablation treatment radiologic imaging diagnoses of the cystic lesions included SCA and epidermoid cyst, we could not find definite histologic evidence of some of the cysts due to lack of lining epithelial cells.

As mentioned earlier, a few histologic features have been identified in resected pancreatic cysts after ablation $[13,14$, 20, 24-26]. Although epithelial denudation, atrophy, and fibrosis have been described in previous studies [14, 20,24], other features such as histiocytic aggregation, marked stromal hyalinization, and diffuse egg shell-like calcification along the cystic wall have not. Interestingly, except for diffuse egg shell-like calcification along the cystic wall, many histologic findings overlapped with those of pancreatic 


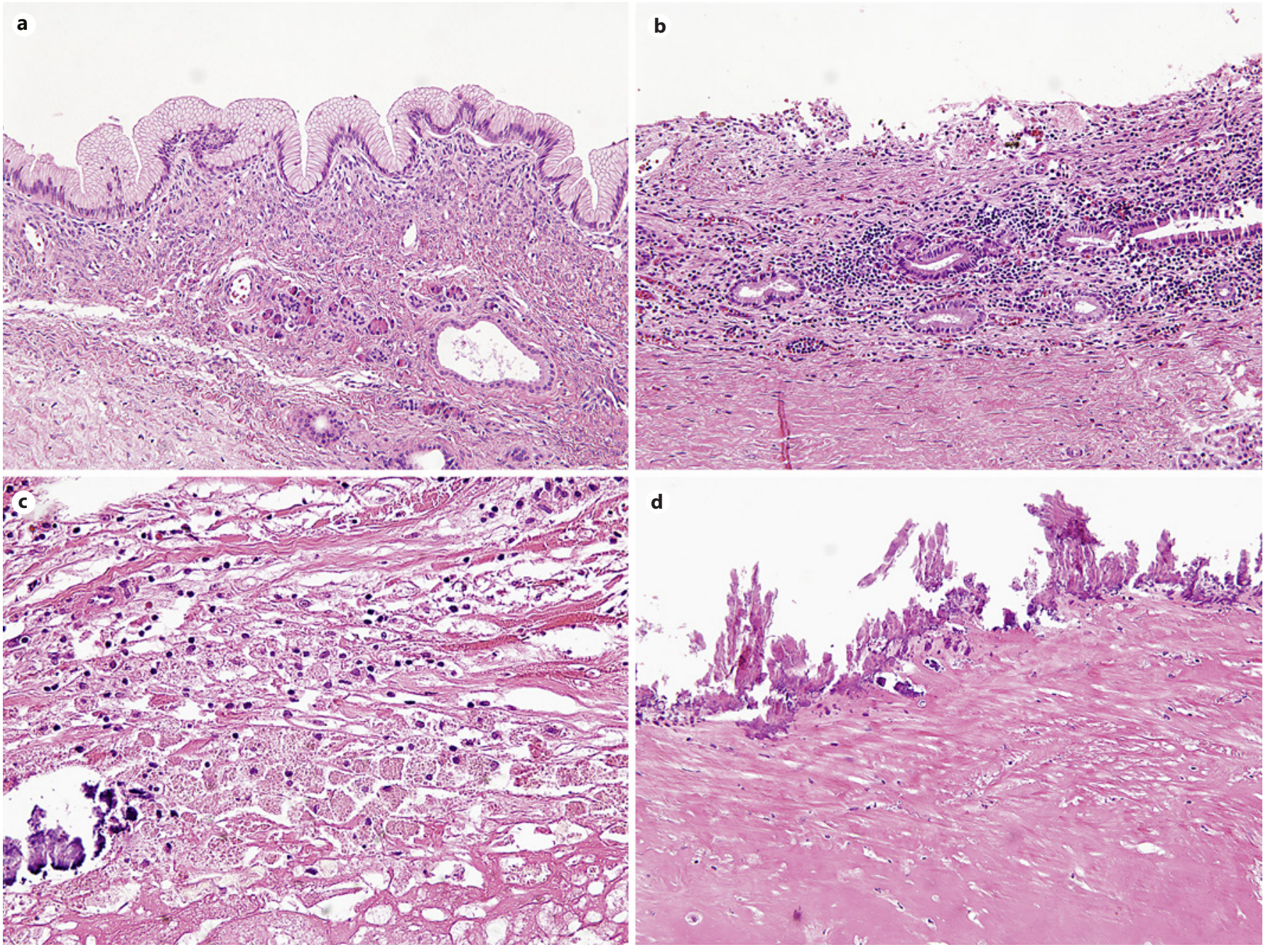

Fig. 2. Representative microscopic images of commonly observed histologic features of pancreatic cyst after ablation therapy. a Residual mucinous lining epithelium with ovarian-type stroma $(\times 200)$. b Moderate stromal lymphoplasmacytic infiltration $(\times 200)$. c Foamy histiocytic aggregation with calcification $(\times 400)$. d Diffuse calcification and stromal hyalinization along the cystic wall $(\times 200)$.

pseudocysts, including moderate chronic active inflammation, histiocytic aggregation, marked stromal hyalinization, granulation tissue formation, hemorrhage, necrotic tissue with brown pigmentation, cholesterol cleft, and fat necrosis. Therefore, residual lining epithelia, ovarian-type stroma, and diffuse egg shell-like calcification along the cystic wall are the only features that can differentiate pancreatic cysts after EUS-guided ablation therapy from pseudocysts. Indeed, in some cases, the patients' history of ablation therapy was not provided at the time of pathology diagnosis. Therefore, the original pathological diagnosis of those cases was a pancreatic pseudocyst.

In conclusion, diffuse egg shell-like calcification along the pancreatic cystic walls with focal residual lining epi- thelia and/or ovarian-type stroma were characteristics of pancreatic cysts after ablation therapies with ethanol and/ or paclitaxel injection which differentiate them from pseudocysts. Understanding these histologic features will be helpful for a precise pathological diagnosis of pancreatic cystic tumor after ablation therapies, even without knowing the patient's history of cystic ablation therapy.

\section{Acknowledgements}

This work was presented in part at the 108th Annual Meeting of the United States and Canadian Academy of Pathology, March 16-21, 2019 in National Harbor, MD, USA. 



Fig. 3. Representative microscopic images of less commonly observed histologic features pancreatic cyst after ablation therapy. a Necrotic tissue with brown pigments with numerous neutrophils $(\times 200)$. b Cholesterol left $(\times 200)$. c Hemorrhage $(\times 40)$. d Fat necrosis in peripancreatic soft tissue $(\times 200)$.

\section{Statement of Ethics}

This study was approved from the Institutional Review Board (approval number, 2013-0618) with waiver of patient consents.

\section{Conflict of Interest Statement}

The authors have no conflicts of interest to declare.

\section{Funding Sources}

This research was supported by the Basic Science Research Program through the National Research Foundation of Korea (NRF) funded by the Ministry of Science, ICT, and Future Planning (NRF-2012R1A1A2003360).

\section{Author Contributions}

Concepts and design: S.-M.H.; definition of intellectual contents: S.-Y.J. and S.-M.H.; literature search and data acquisition: S.A., Y.-N.S., and S.J.K.; clinical studies: D.-W.S.; data analysis: S.A. and S.-Y.J.; statistical analysis: S.A.; manuscript preparation: S.A.; manuscript editing and review: S.-Y.J. and S.-M.H. All authors critically read and approved the manuscript.

\section{Data Availability Statement}

The raw data supporting the conclusions of this article will be made available by the authors. 


\section{References}

1 Law JK, Hruban RH, Lennon AM. Management of pancreatic cysts: a multidisciplinary approach. Curr Opin Gastroenterol. 2013; 29(5):509-16.

2 Laffan TA, Horton KM, Klein AP, Berlanstein B, Siegelman SS, Kawamoto S, et al. Prevalence of unsuspected pancreatic cysts on MDCT. AJR Am J Roentgenol. 2008;191(3): 802-7.

3 de Jong K, Nio CY, Hermans JJ, Dijkgraaf MG, Gouma DJ, van Eijck CH, et al. High prevalence of pancreatic cysts detected by screening magnetic resonance imaging examinations. Clin Gastroenterol Hepatol. 2010; 8(9):806-11.

4 Chang YR, Park JK, Jang JY, Kwon W, Yoon $\mathrm{JH}, \mathrm{Kim} \mathrm{SW}$. Incidental pancreatic cystic neoplasms in an asymptomatic healthy population of 21,745 individuals: large-scale, singlecenter cohort study. Medicine. 2016;95(51): e5535.

5 Spinelli KS, Fromwiller TE, Daniel RA, Kiely JM, Nakeeb A, Komorowski RA, et al. Cystic pancreatic neoplasms: observe or operate. Ann Surg. 2004;239(5):651-9; discussion 7-9.

6 Zerboni G, Signoretti M, Crippa S, Falconi M, Arcidiacono PG, Capurso G. Systematic review and meta-analysis: prevalence of incidentally detected pancreatic cystic lesions in asymptomatic individuals. Pancreatology. 2019;19(1):2-9.

7 Attila T, Adsay V, Faigel DO. The efficacy and safety of endoscopic ultrasound-guided ablation of pancreatic cysts with alcohol and paclitaxel: a systematic review. Eur J Gastroenterol Hepatol. 2019;31(1):1-9.

8 Jun SY, Hong SM. Nonductal pancreatic cancers. Surg Pathol Clin. 2016;9(4):581-93.

9 Goh BK, Tan YM, Cheow PC, Chung YF, Chow PK, Wong WK, et al. Cystic lesions of the pancreas: an appraisal of an aggressive resectional policy adopted at a single institution during 15 years. Am J Surg. 2006;192(2):14854.
10 Allen PJ, D’Angelica M, Gonen M, Jaques DP, Coit DG, Jarnagin WR, et al. A selective approach to the resection of cystic lesions of the pancreas: results from 539 consecutive patients. Ann Surg. 2006;244(4):572-82.

11 Horvath KD, Chabot JA. An aggressive resectional approach to cystic neoplasms of the pancreas. Am J Surg. 1999;178(4):269-74.

12 Khashab MA, Shin EJ, Amateau S, Canto MI, Hruban RH, Fishman EK, et al. Tumor size and location correlate with behavior of pancreatic serous cystic neoplasms. Am J Gastroenterol. 2011;106(8):1521-6.

13 Park JK, Song BJ, Ryu JK, Paik WH, Park JM, Kim J, et al. Clinical outcomes of endoscopic ultrasonography-guided pancreatic cyst ablation. Pancreas. 2016;45(6):889-94.

14 Oh HC, Seo DW, Song TJ, Moon SH, Park DH, Soo Lee S, et al. Endoscopic ultrasonography-guided ethanol lavage with paclitaxel injection treats patients with pancreatic cysts. Gastroenterology. 2011;140(1):172-9.

15 Oh HC, Seo DW, Lee TY, Kim JY, Lee SS, Lee SK, et al. New treatment for cystic tumors of the pancreas: EUS-guided ethanol lavage with paclitaxel injection. Gastrointest Endosc. 2008;67(4):636-42.

16 Gelczer RK, Charboneau JW, Hussain S Brown DL. Complications of percutaneous ethanol ablation. J Ultrasound Med. 1998; 17(8):531-3.

17 Caillol F, Poincloux L, Bories E, Cruzille E, Pesenti C, Darcha C, et al. Ethanol lavage of 14 mucinous cysts of the pancreas: a retrospective study in two tertiary centers. Endosc Ultrasound. 2012;1(1):48-52.

18 DeWitt J, McGreevy K, Schmidt CM, Brugge WR. EUS-guided ethanol versus saline solution lavage for pancreatic cysts: a randomized, double-blind study. Gastrointest Endosc. 2009;70(4):710-23.

19 DiMaio CJ, DeWitt JM, Brugge WR. Ablation of pancreatic cystic lesions: the use of multiple endoscopic ultrasound-guided ethanol lavage sessions. Pancreas. 2011;40(5):664-8.
20 Gan SI, Thompson CC, Lauwers GY, Bounds BC, Brugge WR. Ethanol lavage of pancreatic cystic lesions: initial pilot study. Gastrointest Endosc. 2005;61(6):746-52.

21 DeWitt JM, Al-Haddad M, Sherman S, LeBlanc J, Schmidt CM, Sandrasegaran K, et al. Alterations in cyst fluid genetics following endoscopic ultrasound-guided pancreatic cyst ablation with ethanol and paclitaxel. Endoscopy. 2014;46(6):457-64.

22 Moyer MT, Dye CE, Sharzehi S, Ancrile B, Mathew A, McGarrity TJ, et al. Is alcohol required for effective pancreatic cyst ablation? The prospective randomized CHARM trial pilot study. Endosc Int Open. 2016;4(5): E603-7.

23 Moyer MT, Sharzehi S, Mathew A, Levenick JM, Headlee BD, Blandford JT, et al. The safety and efficacy of an alcohol-free pancreatic cyst ablation protocol. Gastroenterology. 2017;153(5):1295-303.

24 Oh HC, Seo DW, Kim SC, Yu E, Kim K, Moon $\mathrm{SH}$, et al. Septated cystic tumors of the pancreas: is it possible to treat them by endoscopic ultrasonography-guided intervention? Scand J Gastroenterol. 2009;44(2):242-7.

25 Choi JH, Seo DW, Song TJ, Park DH, Lee SS, Lee SK, et al. Long-term outcomes after endoscopic ultrasound-guided ablation of pancreatic cysts. Endoscopy. 2017;49(9):866-73.

26 Kim KH, McGreevy K, La Fortune K, Cramer H, DeWitt J. Sonographic and cyst fluid cytologic changes after EUS-guided pancreatic cyst ablation. Gastrointest Endosc. 2017; 85(6):1233-42.

27 Rowinsky EK, Donehower RC. Paclitaxel (taxol). N Engl J Med. 1995;332(15):1004-14.

28 An S, Kim MJ, Kim SJ, Sung YN, Kim YW, Song KB, et al. Multiple KRAS mutations in the non-mucinous epithelial lining in the majority of mucinous cystic neoplasms of the pancreas. Histopathology. 2019;75(4):55967. 